\title{
Subcritical flow past a circular cylinder surrounded by a porous layer
}

\author{
M. P. Sobera, ${ }^{\text {a) }}$ C. R. Kleijn, ${ }^{\text {b) }}$ and H. E. A. Van den Akker \\ Department of Multi-Scale Physics, Delft University of Technology, Prins Bernhardlaan 6, \\ 2628BW Delft, The Netherlands
}

(Received 2 June 2005; accepted 3 November 2005; published online 31 March 2006)

\begin{abstract}
A study of the flow at subcritical $\mathrm{Re}=3900$ around a circular cylinder, surrounded at some fixed small distance by a porous layer with a hydraulic resistance typical for that of textile materials, has been performed by means of direct numerical simulations. The flow in the space between the porous layer and the solid cylinder was found to be laminar and periodic, with a frequency locked to that of the vortex shedding in the wake behind the cylinder. Time averaged flow velocities underneath the porous material were in good agreement with experimental data from laser Doppler anemometry. (c) 2006 American Institute of Physics. [DOI: 10.1063/1.2189284]
\end{abstract}

Due to its relevance for many technological applications, the turbulent flow past a circular cylinder has been extensively studied by numerous authors, ${ }^{1}$ and is still a topic of current investigations. The numerical simulation of such flows remains to be a challenging task. Breuer ${ }^{2}$ presented results of large eddy simulation (LES) of high Reynolds number $(\mathrm{Re}=140000)$ flow past a circular cylinder. Travin and co-workers ${ }^{3}$ reported results on detached-eddy simulations of the flow at $\mathrm{Re}=50000,140000$, and $3 \times 10^{6}$. Singh and Mittal ${ }^{4,5}$ calculated the flow past a circular cylinder for $\mathrm{Re}=100$ to $10^{7}$ by means of two-dimensional (2D) LES. In literature, special attention is paid to the flow around a cylinder at subcritical $\mathrm{Re}=3900$, both through numerical (e.g., Refs. 6-10) and experimental studies (e.g., Refs. 11 and 12).

Embroidering on the studies mentioned above, the present paper deals with the flow configuration depicted in Fig. 1. It consists of a solid circular cylinder of radius $r_{s}=0.5 D_{s}$, surrounded at some small and fixed distance $\delta_{g}=r_{c}-\left(r_{s}+\delta_{c}\right)$ by a thin porous layer of thickness $\delta_{c}$ and outer radius $r_{c}=0.5 D_{c}$ and with a hydraulic resistance typical for that of textile materials. This configuration is of practical relevance in for instance filtering and separation technology and as a standard reference configuration for testing protective and insulating properties of clothing materials. ${ }^{13} \mathrm{De}-$ pending on its hydraulic resistance, the general characteristics of the outer flow around the porous layer are expected to be similar in nature to those of the flow around a solid cylinder. However, not much is known about the interaction between the unsteady, turbulent flow outside the porous cylinder and flow inside the fluid gap between the porous and the solid cylinder. An experimental study of the velocity field around a fabric covered cylinder has been reported by Watanabe and co-workers, ${ }^{14}$ who studied the influence of the porous layer permeability on the velocity of the flow underneath the porous layer. Experimental results on heat transfer to a fabric covered cylinder have been reported by Kind and co-workers. ${ }^{15}$ Gibson ${ }^{16}$ presented numerical computations of the air flow and heat transfer to a fabric-covered cylinder. A

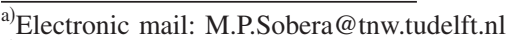

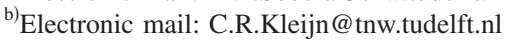

numerical study of free and mixed convection around a cylinder surrounded by porous material, focusing on situations where there is no or very little external air flow, was presented by Bo and Nakijama. ${ }^{17}$

In a previous paper, ${ }^{18}$ we reported simulations of the subcritical turbulent fluid flow, heat and mass transfer for this geometry by means of the Reynolds averaged Navier Stokes (RANS) approach, as well as scaling rules for the flow characteristics as a function of the Reynolds number, the Darcy number-which is a measure for the hydraulic resistance of the porous layer-and the geometric ratios $\mathrm{Ig}=\delta_{g} / D_{c}$ and $\mathrm{Ic}=\delta_{c} / D_{c}$. The RANS approach raised several questions regarding the nature of the flow underneath the porous layer, especially regarding laminarization and (un)steadiness. In order to address the questions of laminarization and (un)steadiness of the flow underneath the porous layer in a geometry such as in Fig. 1, the aim of the present work was to investigate the interaction between the periodic, subcritical turbulent flow outside the outer cylinder, and the flow inside the fluid gap between the two cylinders. For this, we used direct numerical simulations (DNS). As in most DNS studies of the flow around a solid cylinder, we chose a Reynolds number, based on the free stream velocity $u_{\infty}$ and the porous cylinder diameter $D_{c}$, of $\mathrm{Re}=3900$. The thickness ratio of the fluid gap was set as $\operatorname{Ig}=\delta_{g} / D_{c}=5 \times 10^{-2}$. In the porous material, the Navier Stokes equations were extended with an additional momentum sink term according to Darcy's law. For the hydrodynamic permeability $k$ and thickness $\delta_{c}$ of the porous layer, values of practical interest for applications in testing clothing material have been used, leading to $\mathrm{Da}=k / \delta_{c}^{2}$ $=2 \times 10^{-3}$ and $\mathrm{Ic}=5 \times 10^{-3}$.

The three-dimensional computational domain was similar to that used by Ma et al. ${ }^{9}$ and Tremblay ${ }^{10}$ in their studies of the flow around a solid cylinder. At the inflow $\left(x=-5 D_{c}\right)$ plane, a potential flow of constant velocity $u_{\infty}$ has been imposed, and at the outflow $\left(x=14 D_{c}\right)$ plane a convective boundary condition was used. Periodic boundary conditions were imposed at the $y=4 D_{c}$ and $y=-4 D_{c}$ planes. The spanwise length of the simulated cylinder was $\pi D_{c}$, with periodic boundaries imposed on the sides. Since we treated the porous layer as a fluid zone, no boundary conditions had 


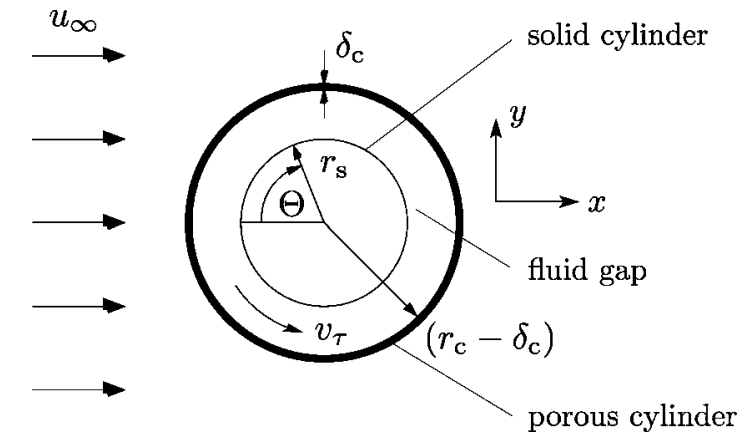

FIG. 1. Schematic description of the problem.

to be imposed on the inner and outer interfaces of the porous layer. We applied an unstructured, nonuniform finite volume quadrilateral grid, with local grid refinement in the vicinity of the cylinder and in the near wake region, which was boundary fitted to the solid cylinder surface and the inner and outer porous layer surfaces. There were three layers of body fitted cells within the porous layer thickness of $0.005 D_{c}$. The momentum sink term was set to be uniform within each grid cell, based on the velocity in that cell. The dimensionless distance from the first grid point to the cylinder surface was assured to be $y^{+}<1$. Similar to the grid used by Tremblay, ${ }^{10}$ the $\Delta x$ and $\Delta y$ grid spacing size in the very near wake region were approximately $0.008 D_{c}$. In the far field a lower grid resolution was used. The $\Delta z$ grid spacing was constant over the entire domain as $\Delta z=0.031 D_{c}$. The number of grid cells outside the porous layer was approximately $5 \times 10^{6}$. In the fluid gap between the cylinder and the porous layer there were an additional $1.5 \times 10^{6}$ grid cells. A second-order central differencing scheme was used for spatial discretization of the flow equations. For time discretization, a second-order implicit scheme was used. Simulations were run with a constant time step $\Delta t=0.001 D_{c} / u_{\infty}$. The simulations were carried our for nine vortex cycles, after the flow had developed, discarding the transient development phase. Although probably insufficient for achieving a proper statistical description of the outer flow, this was sufficient for obtaining a proper description of the low frequency flow

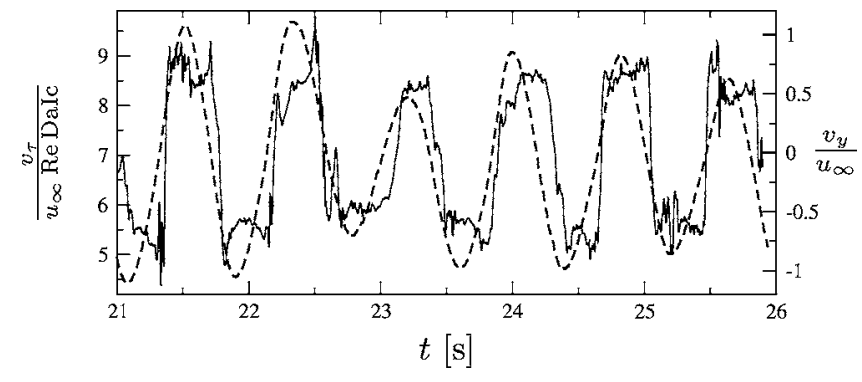

FIG. 2. Time history of the transverse velocity component $v_{y}$ in the wake at $x / D=3$ and $y / D=0$ (solid line) and the tangential velocity component $v_{\tau}$ inside the fluid gap at $\Theta=45^{\circ}$ and $\left(r-r_{s}\right) /\left(r_{s}-r_{c}\right)=0.5$ (dashed line). The applied scaling for the tangential velocity has been reported in Ref. 18

inside the fluid gap. To make sure that the chosen grid resolution was sufficient for DNS of this complicated flow, the applied numerical scheme and computational mesh have been tested extensively in a separate study, ${ }^{19}$ in which results obtained for the flow around a solid cylinder at $\mathrm{Re}=3900$ have been found to compare excellently to DNS and experimental data from literature.

As expected for the relatively low permeability studied here, the main characteristics of the flow around the porous cylinder at $\mathrm{Re}=3900$-based on the porous cylinder diameter-were very similar to those of the flow around a solid cylinder at the same Re. ${ }^{19}$ The Strouhal number for the porous and solid cylinder was 0.212 and 0.218 , respectively. The length of the recirculation bubble is somewhat shorter for the porous case: $L_{B} / D=1.12$, as compared to 1.32 for the solid cylinder. ${ }^{19}$ The flow inside the fluid gap (i.e. in the space between the outer porous layer and the inner solid cylinder) was found to be laminar and periodic. The dynamics of this inner flow is due to the vortex shedding phenomena in the wake of the outer flow. In Fig. 2, a comparison between the velocity at a point inside the fluid gap and the velocity at a specific location in the wake is presented. We can observe that the inner flow exhibits a periodic behavior and that its frequency is locked to the frequency of the wake oscillations due to the vortex shedding phenomenon. However, only the low Strouhal frequency is pronouncedly
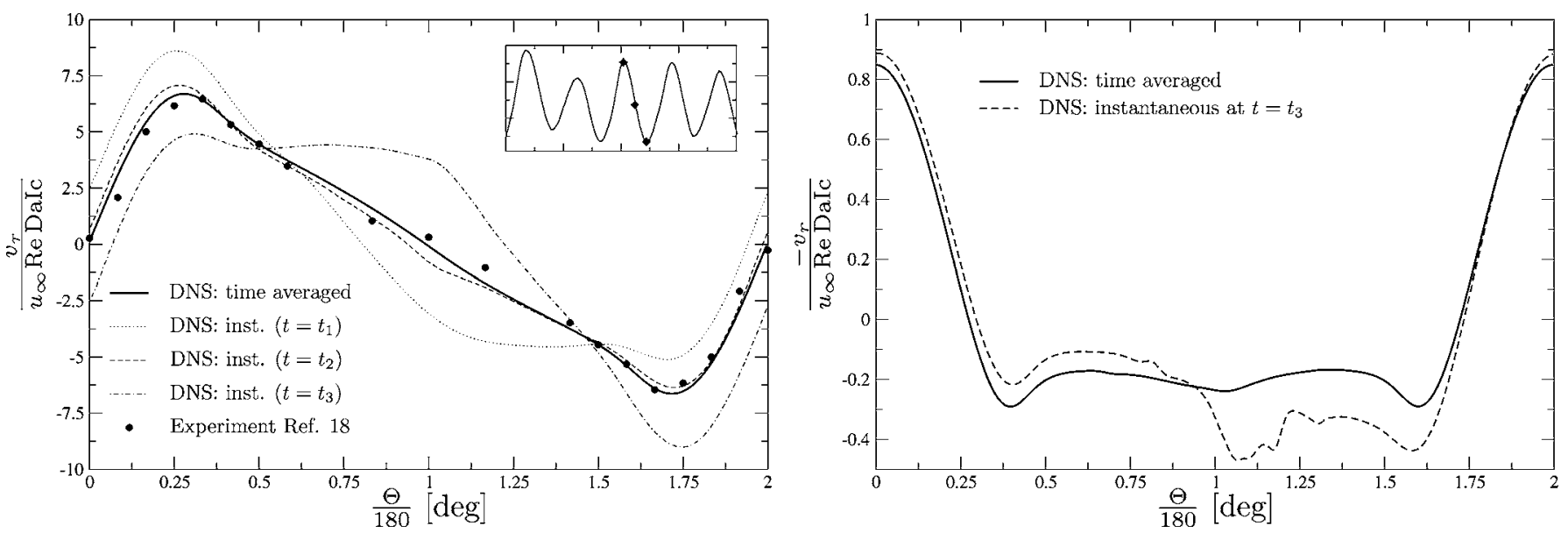

FIG. 3. Tangential velocity $v_{\tau}$ at the fluid gap centerline (left) and the radial velocity $v_{r}$ through the porous layer (right), both as a function of angle. The applied scaling for the velocities has been reported in Ref. 18. 
present in the flow inside the fluid gap, whereas the higher frequencies seem to be filtered out. Please note, that the magnitude of the flow velocity inside the fluid gap is orders of magnitude smaller than that of the velocity in the wake.

Figure 3 presents the tangential velocity component along the centerline at $\left(r-r_{s}\right) /\left(r_{c}-r_{s}\right)=0.5$ inside the fluid gap (left) and the radial velocity component through the porous layer (right), both as a function of angular coordinate $\Theta$. Both velocities are scaled with the free stream velocity, with $\mathrm{Re}$ and with the dimensionless hydraulic resistance DaIc $=k /\left(\delta_{c} D_{c}\right)$. Details on the scaling rule used here are reported in Ref. 18. In the left-hand part of Fig. 3, the timeaveraged dimensionless tangential velocity is presented, together with three of its instantaneous time realizations. These time realizations were taken at instances corresponding to the maximal, intermediate, and minimal value, respectively, of the tangential velocity at $\Theta=45^{\circ}$. The inset figure is a simplified representation of the periodic inner flow behavior from Fig. 2, with the three time realizations indicated by black dots. The velocity distribution in the fluid gap at different time instances confirms the periodic nature of that flow. The two extreme time realizations are symmetric with respect to $\Theta=180^{\circ}$. The velocity profile at the intermediate time instant is very similar to the time-averaged velocity profile. The latter is in very good agreement with our timeaveraged experimental data obtained by laser Doppler anemometry. ${ }^{18}$ In the right-hand part of Fig. 3, the radial velocity, i.e., the velocity component perpendicular to the porous layer, is presented as a function of the angular coordinate. The time-averaged radial velocity component is presented together with an instantaneous time realization at $t=t_{3}$. For $\Theta<50^{\circ}$ and $\Theta>310^{\circ}$ the radial velocity component is positive, meaning that the fluid flows from the outer area into the fluid gap. For $50^{\circ}<\Theta<310^{\circ}$ it flows from the fluid gap into the outer area. As to be expected, the long time-averaged radial velocity is symmetrical with respect to $\Theta=180^{\circ}$, and the amount of fluid flowing towards the fluid gap is equal to the amount of fluid leaving the fluid gap. The small asymmetry visible in the time-averaged curve in the right-hand side of Fig. 3 is probably due to an insufficiently long averaging time (nine vortex shedding cycles only). Analyzing the instantaneous radial velocity distribution at $t=t_{3}$,

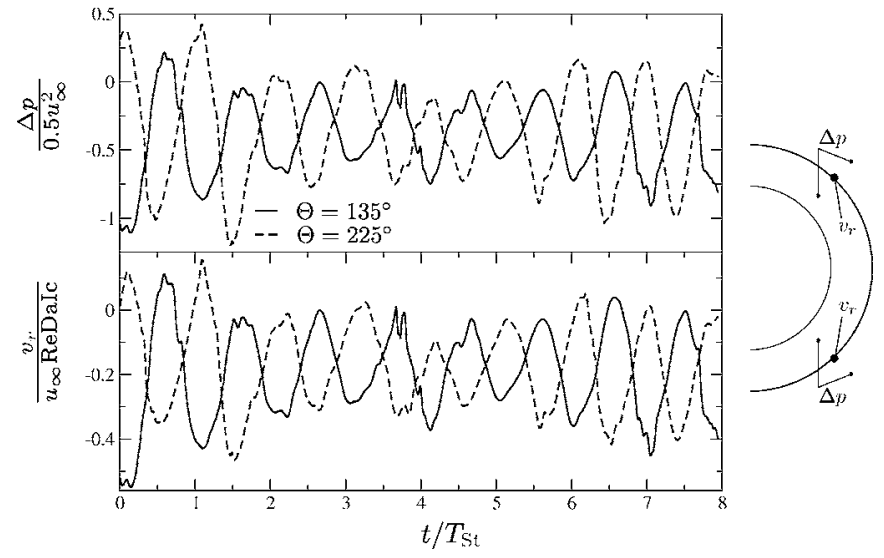

FIG. 4. Upper left-hand figure: Pressure difference between the wake and the fluid gap as a function of time, in two monitored points in the upper downstream $\left(\Theta=135^{\circ}\right.$, solid line $)$ and lower downstream $\left(\Theta=225^{\circ}\right.$, dashed line) parts of the cylinder. Lower left-hand figure: Velocity through the porous layer (in the same two points, as a function of time). Right-hand figure: Schematic illustration of the locations of the two monitored points.

we can explain the wavy behavior of the flow inside the fluid gap. The instantaneous radial velocity distribution is nonsymmetrical due to the countercurrent vortex shedding at the downstream surface of the porous layer, which causes a pressure difference between the top and the bottom part of the cylinder. In the presented graph, the outgoing fluid velocity is higher at the bottom part $\left(\Theta>180^{\circ}\right)$ of the porous cylinder, which is due the vortex being formed and shed at this part.

Figure 4 shows the strong correlation between the instantaneous pressure difference over the porous material (nondimensionalized with the free stream dynamic pressure), and the radial velocity through the porous material (nondimensionalized as in previous figures; see Ref. 18), at two locations in the downstream top and bottom part of the cylinder. These two points have been indicated in the pictogram at the right-hand side of the figure and are located at $\Theta=135^{\circ}$ and $\Theta=225^{\circ}$, respectively. As mentioned above, the periodic behavior of the flow inside the fluid gap is due to the vortex shedding at the back surface of the obstacle. The radial velocity component exhibits strong correlation to the
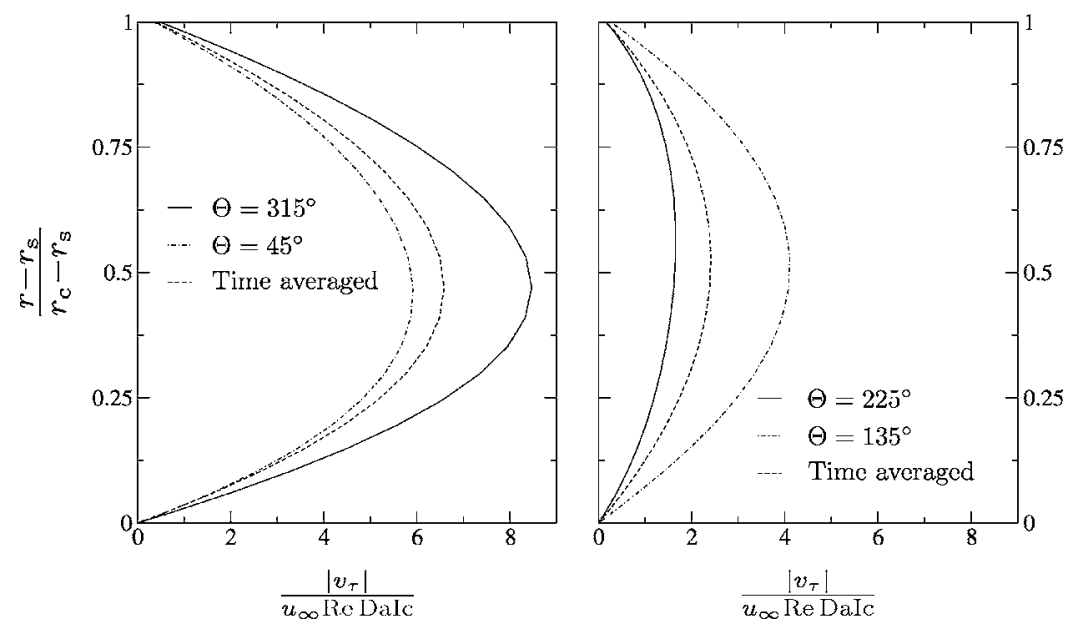

FIG. 5. The time averaged and the instantaneous tangential velocity profiles inside the fluid gap at different angular position: $45^{\circ}$ and $315^{\circ}$ (left) and $135^{\circ}$ and $225^{\circ}$ (right). 
pressure difference $\Delta p=p_{2}-p_{1}$ between the fluctuating pressure $p_{2}$ in the wake and the fluctuating pressure $p_{1}$ inside the fluid gap. Taking into account the fact that the pressure variations inside the fluid gap are much smaller than those in the wake due to the large pressure drop over the porous layer $\left(p_{1} \ll p_{2}\right)$, we can conclude that the velocity through the porous layer directly correlates to the pressure fluctuations in the wake.

Figure 5 shows profiles of the absolute value of the (scaled) tangential velocity component $\left|v_{\tau}\right|$ inside the fluid gap. Instantaneous profiles at four different locations $\left(\Theta=45^{\circ}, 135^{\circ}, 225^{\circ}\right.$, and $\left.315^{\circ}\right)$ at one instant in time are presented together with the time-averaged ones. The time instant presented here corresponds to $t=t_{3}$ from Fig. 3, i.e., the time instant of maximal $\left|v_{\tau}\right|$ at $\Theta=315^{\circ}$. At this time instant, due to the interaction with the wake vortices, most of the entering fluid passes through the lower half of the fluid gap, as can be seen from the larger velocities at $\Theta=315^{\circ}$ than at $\Theta=45^{\circ}$. Of course, due to the long-term symmetry, the time-averaged velocity profiles for $\Theta=45^{\circ}$ and $\Theta=315^{\circ}$ are identical, as well as those for $\Theta=135^{\circ}$ and $\Theta=225^{\circ}$. Please note that in the upper part of the fluid gap the tangential velocity is of the same order of magnitude at both monitored locations $\left(\Theta=45^{\circ}\right.$ and $\left.135^{\circ}\right)$. Only a little fluid has left the gap between these two locations. In the lower part, the tangential velocity is significantly higher at $\Theta=315^{\circ}$ than at $\Theta=225^{\circ}$. This imbalance of the tangential momentum confirms that most of the flow leaves the fluid gap in the space between these locations. The general shape of the tangential velocity profiles illustrates the laminar character of the flow inside the fluid gap. The nonzero value of $v_{\tau}$ at $\left(r-r_{s}\right) /\left(r_{c}-r_{s}\right)=1$ is due the nonzero permeability of the outer wall, allowing for tangential flow inside this wall. In the upstream locations $\left(\Theta=45^{\circ}\right.$ and $\left.\Theta=315^{\circ}\right)$, where the fluid is entering the gap from the outside, the tangential velocity maximum is pushed inward and occurs for $\left(r-r_{s}\right) /\left(r_{c}-r_{s}\right)<0.5$. Similarly, in the downstream locations, where air is flowing out from the fluid gap, the tangential velocity maximum is located at $\left(r-r_{s}\right) /\left(r_{s}-r_{g}\right)>0.5$.

In conclusion, we found that in the case of a subcritical turbulent flow around a cylinder surrounded by a porous layer with a hydraulic resistance typical for that of textile materials, the flow underneath that porous layer is laminar and periodic, with the frequency locked to the Strouhal frequency of vortex shedding. The velocity through - and as a consequence the velocity underneath - the porous layer is driven by the pressure oscillations in the wake behind the obstacle.

The authors would like to thank Professor K. Hanjalic and Dr. B. Niceno for allowing them to use their T-Rex code. This research was performed in the framework of a project supported and financed by the Prins Maurits Laboratory of the Netherlands Organization for Applied Scientific Research (TNO-PML). This work was sponsored by the Netherlands National Computing Facilities for the use of supercomputer facilities, with financial support from the Netherlands Organization for Scientific Research (NWO).

${ }^{1}$ C. H. K. Williamson, "Vortex dynamics in the cylinder wake," Annu. Rev. Fluid Mech. 28, 477 (1996).

${ }^{2}$ M. Breuer, "A challenging test case for large eddy simulation: high Reynolds number circular cylinder flow," Int. J. Heat Fluid Flow 21, 648 (2000).

${ }^{3}$ A. Travin, M. Shur, M. Strelets, and P. Spalart, "Detached-eddy simulations past a circular cylinder," Flow, Turbul. Combust. 63, 293 (2000).

${ }^{4}$ S. P. Singh and S. Mittal, "Flow past a cylinder: shear layer instability and drag crisis," Int. J. Numer. Methods Fluids 47, 75 (2005).

${ }^{5}$ S. P. Singh and S. Mittal, "Energy spectra of flow past a circular cylinder," Int. J. Comput. Fluid Dyn. 18, 671 (2004).

${ }^{6} \mathrm{M}$. Breuer, "Numerical and modeling influences on large eddy simulations for the flow past a circular cylinder," Int. J. Heat Fluid Flow 19, 512 (1998).

${ }^{7}$ J. Franke and W. Frank, "Large eddy simulation of the flow past a circular cylinder at Re-D=3900," J. Wind. Eng. Ind. Aerodyn. 90, 1191 (2002).

${ }^{8}$ A. G. Kravchenko and P. Moin, "Numerical studies of flow over a circular cylinder at Re-D=3900," Phys. Fluids 12, 403 (2000).

${ }^{9}$ X. Ma, C. S. Karamanos, and G. E. Karniadakis, "Dynamics and lowdimensionality of a turbulent near wake," J. Fluid Mech. 410, 29 (2000).

${ }^{10} \mathrm{~F}$. Tremblay, "Direct and large-eddy simulation of flow around a circular cylinder at subcritical Reynolds numbers," Ph.D. thesis, Munich University of Technology, Germany, 2001.

${ }^{11} \mathrm{P}$. Beaudan and P. Moin, "Numerical experiments on the flow past a circular cylinder at sub-critical Reynolds number," Technical Report TF-62, Thermoscience Division, Department of Mechanical Engineering, Stanford University, 1994.

${ }^{12} \mathrm{~L}$. Ong and J. Wallace, "The velocity field of the turbulent very near wake of a circular cylinder," Exp. Fluids 20, 441 (1996).

${ }^{13}$ L. F. G. Geers, "Developments in modeling NBC protective clothing," Technical Report PML2004-A122, TNO-PML, The Netherlands, 2004.

${ }^{14}$ T. Watanabe, T. Kato, and Y. Kamata, "The velocity distribution in the inner flow field around a clothed cylinder," Sen'i Gakkaishi 47, 271 (1991).

${ }^{15}$ R. J. Kind, C. A. Jenkis, and C. A. Broughton, "Measurement of windinduced heat transfer through permeable cold-weather clothing," Cold Regions Sci. Technol. 23, 305 (1995).

${ }^{16} \mathrm{P}$. W. Gibson, "Review of numerical modeling of convection, diffusion, and phase change in textiles," in Computational Technologies for Fluid/ Thermal/Structural/Chemical Systems with Industrial Applications, edited by C. R. Kleijn and S. Kawano (ASME, New York, 1999), Vol. 397-2, pp. 117-126.

${ }^{17} \mathrm{Q}$. Bo and T. Nakijama, "Natural and mixed convection around a cylinder enclosed with porous media-a numerical study on comfort of clothed human being," in Computational Technologies for Fluid/Thermal/ Structural/Chemical Systems with Industrial Applications, edited by C. R. Kleijn and V. Kudriavtsev (ASME, New York, 2001), Vol. 424-2, pp. 177-183.

${ }^{18}$ M. P. Sobera, C. R. Kleijn, P. Brasser, and H. E. A. van den Akker, "Convective heat and mass transfer to a cylinder sheathed by a porous layer," AIChE J. 49, 3018 (2003).

${ }^{19}$ M. P. Sobera, C. R. Kleijn, P. Brasser, and H. E. A. van den Akker, "Hydrodynamics of the flow around a circular cylinder sheathed by a porous layer," in Proceedings of the 2004 ASME Heat Transfer/Fluids Engineering Summer Conference, Charlotte, NC (ASME, New York, 2004) (CD). 